\title{
The Impact of Diabetes on the Risk of Prostate Cancer Development according to Body Mass Index: A 10-year Nationwide Cohort Study
}

\author{
Jin Bong Choi ${ }^{1,3}$, Hyong Woo Moon', Young Hyun Park ${ }^{1,3}$, Woong Jin Bae ${ }^{1}$, Hyuk Jin Cho ${ }^{1}$, Sung-Hoo \\ Hong1,3, Ji Youl Lee ${ }^{1,3}$, Sae Woong Kim¹, Kyung-Do Han², and U-Syn Ha',3凶 \\ 1. Department of Urology, Seoul St. Mary's Hospital, College of Medicine, The Catholic University of Korea, Seoul, Republic of Korea; \\ 2. Department of Biostatistics, College of Medicine, The Catholic University of Korea, Seoul, Republic of Korea; \\ 3. The Cancer Research Institute, The Catholic University of Korea, Seoul, Republic of Korea. \\ $\triangle$ Corresponding author: U-Syn Ha, MD, PhD, Department of Urology, Seoul St. Mary's Hospital, College of Medicine, The Catholic University of Korea, 222, \\ Banpo-daero, Seocho-gu, Seoul, 06591, Republic of Korea. Tel: +82-2-2258-6224, Fax: +82-2-599-7839, e-mail address: ushamd@catholic.ac.kr
}

() Ivyspring International Publisher. Reproduction is permitted for personal, noncommercial use, provided that the article is in whole, unmodified, and properly cited. See http://ivyspring.com/terms for terms and conditions.

Received: 2016.05.09; Accepted: 2016.09.04; Published: 2016.10.22

\begin{abstract}
Purpose: We examined the association between obesity and prostate cancer both with and without diabetic patients included in the analysis using nationally representative data of the Korean population from the National Health Insurance System (NHIS).

Materials and Methods: Of the 424,712 participants who underwent health examinations in 2002-2008, 139,519 men $\geq 40$ years old and without prostate cancer were followed from the beginning of 2002 to the end of 2012. Multivariate adjusted Cox regression analysis was conducted to examine the hazard ratio $(\mathrm{HR})$ and $95 \%$ confidence interval $(\mathrm{Cl})$ for the association between prostate cancer and body mass index (BMI) both with and without diabetes.

Results: The HR for prostate cancer according to the existence of diabetes was stratified by BMI in both age- and multivariable-adjusted models. In the population without diabetes, the HR for prostate cancer significantly increased as BMI increased beyond the reference range in a model adjusted for age and multiple variables; however, the increase in the HR was small. In the population with diabetes, the $\mathrm{HR}$ for prostate cancer significantly increased as BMI increased from $<18.5 \mathrm{~kg} / \mathrm{m}^{2}$ to within the reference range (18.5 to 22.9) in the multivariable-adjusted model. In addition, a marked decrease in HR in the population with BMI of $<18.5 \mathrm{~kg} / \mathrm{m}^{2}$ was seen compared to the reference or higher BMI population.

Conclusion: This population-based study shows the evidence of association between obesity and development of prostate cancer, and the risk increases vary according to the change of BMl category and the existence of diabetes.
\end{abstract}

Key words: Prostate cancer; Obesity; Diabetes.

\section{Introduction}

Prostate cancer is the second most commonly diagnosed malignancy worldwide. Though it is less common in Korea than in the Western Hemisphere, the incidence has sharply increased recently [1]. This trend is most likely explained by increased prevalence of obesity, resulting from lifestyle changes associated with westernization, such as physical inactivity and a greater intake of dietary fat and meat among Koreans.
However, the biological mechanisms linking westernization and increased prostate cancer are still unclear. The only demonstrated risk factors to date that lead to prostate cancer are ethnicity, age, and family history of prostate cancer [2,3].

Recently, multiple epidemiologic studies have suggested that obesity is associated with an increased risk for the development of prostate cancer [4, 5]. 
However, results of individual studies have been somewhat inconsistent, as some have suggested that the association between obesity and prostate cancer may differ by tumor aggressiveness [6-8], and others have reported a protective effect of obesity $[9,10]$.

We thought that these discrepancies might be due to poor representations of study populations and small sample sizes. So, we conducted a nationwide, population-based study to estimate the relationship between obesity and development of prostate cancer with 10 years of follow-up. It is also plausible that diabetes could be an important confounding factor in previous investigations, and there has been no retrospective, large, national cohort study involving population samples stratified on diabetes to our knowledge [11-14].

Therefore, in this study, we examined the association between obesity and prostate cancer in patients both with and without diabetes using nationally representative data of the Korean population.

\section{Materials and Methods}

In Korea, almost all citizens are enrolled in the National Health Insurance System (NHIS) as either an employee or a member of a community. The NHIS contains comprehensive health-related information for approximately $97.1 \%$ of the population in Korea

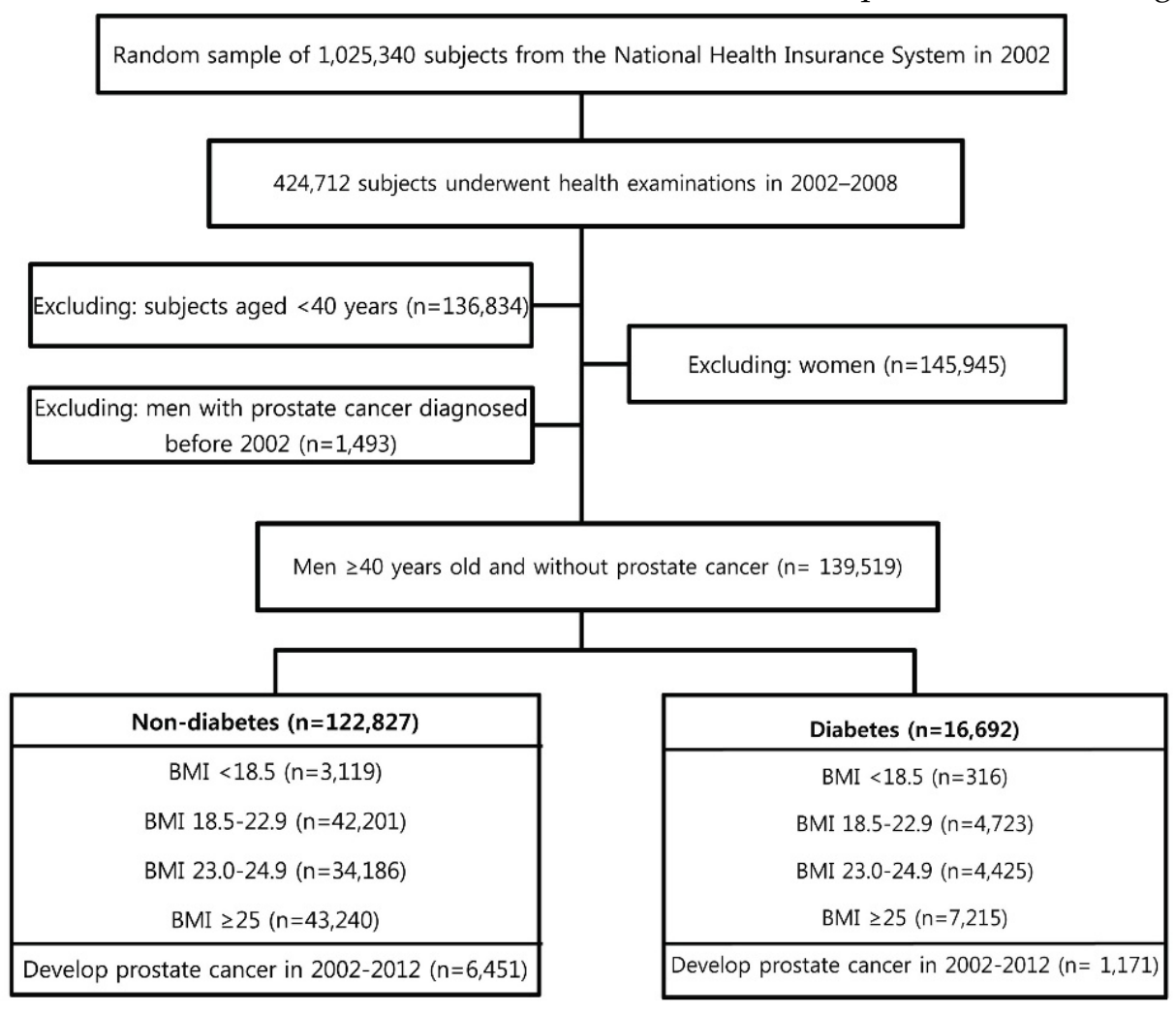

Figure 1. Study design and disposition of subjects. and is composed of an eligibility database, a medical treatment database, a health examination database, and a medical care institution database. A customized database from NHIS includes 1,025,340 subjects that were selected by systematic sampling method to generate a representative sample from a total $46,605,433$ Korean population in 2002. In the present study, sex, birth date, and diagnostic codes based on the International Classification of Diseases, Tenth Revision, Clinical Modification (ICD-10-CM) were retrieved. Diabetes was coded E10-14, and prostate cancer was coded C61. Because the Korean government has enhanced the benefit coverage for four major conditions (cancer, cardiovascular disease, cerebrovascular disease, and rare diseases), virtually all physician-diagnosed cancer patients are registered in the database. To protect the individual's privacy, each patient's identification number was anonymized.

Of the 424,712 participants who underwent health examination at least one time from 2002-2008, those aged $<40$ years $(\mathrm{n}=136,834)$ were excluded because prostate cancer is rare in young men. The information about diabetes and body mass index (BMI) was reported in this health examination. The Korean government has chosen the age of 65 years as the definition of elderly. Thus, we analyzed data for those aged $\geq 40$ years stratified as follows: ages 40-64 and $\geq 65$ years. After excluding women and those with prostate cancer diagnosed before 2002, 139,519 men $\geq 40$ years old and without prostate cancer were followed from the beginning of 2002 to the end of 2012. The events did not occur during the time were censored and Cox regression analysis was used for these censored data. The percentage of missing values was approximately $15 \%$ for the variables. Sensitivity analysis was used to handle these missing data. Figure 1 shows a flowchart for selecting cases for the study. This study was approved by the Institutional Review Board of The Catholic University of Korea (No. KC15RISI0633). Anonymized and de-identified information was used for analysis, and therefore the need for informed consent was waived. 
BMI was calculated as weight in kilograms divided by height in square meters. The Korean Society for the Study of Obesity recommends the use of the following BMI ranges: underweight (under 18.5); normal weight (18.5 to 22.9); overweight (23 to 24.9); and obese (over 25) [15]. Blood pressure was measured while the subject was in a seated position after five minutes of rest. Blood samples were collected after an overnight fasting and measured for serum levels of glucose, total cholesterol, triglycerides, high density lipoprotein cholesterol, and low density lipoprotein cholesterol.

The definition of socioeconomic and lifestyle variables was as follows. Smoking status was categorized into three groups: non-smokers, current smokers who had smoked 100 cigarettes or more in their lifetime, and ex-smokers - those who had smoked in the past but had since quit. Alcohol consumption status was categorized into three groups: non-drinker, those who drank two or three times a month, and those who drank weekly. Exercise status was also divided into three groups: non-exerciser, physical activity performed for at least 30 min less than four times a week, and more than five times a week. Household income was calculated as the family income, adjusting for the number of family members, and divided into Yes (below 5th quintile) and No.

SAS version 9.3 (SAS Institute, Cary, NC, USA) was used for statistical analysis. The data in this study are presented as the mean \pm standard error (SE) or proportions for continuous or categorical variables, respectively. If necessary, a logarithmic transformation was performed to achieve a normal distribution. Multivariate adjusted Cox regression analysis was conducted to examine the hazard ratio $(\mathrm{HR})$ and 95\% confidence interval (CI) for the association between prostate cancer and BMI both with and without diabetes. Calculations were made adjusting for age, smoking status, alcohol consumption, exercise, income, and residential area. A p-value $<0.05$ was considered statistically significant.

\section{Results}

During 1,179,485 person-years of follow-up, 7,622 incident cases of prostate cancer developed between the beginning of 2002 and end of 2012, and the incidence density was 6.5 cases per 1,000 person-years.

\section{Comparison of clinical characteristics according to diagnosis of diabetes}

Table 1 summarizes the general characteristics of the study population and subgroups. Among a total of 139,519 participants, 16,692 (11.96\%) were diagnosed with diabetes, including 3,731 (22.37\%) elderly participants. Mean BMI and total cholesterol were significantly higher in those with diabetes. The proportion of hypertension, dyslipidemia, and prostate cancer was also significantly higher in those with diabetes.

Table 1. Comparison of clinical characteristics according to the presence of diabetes.

\begin{tabular}{|c|c|c|c|c|}
\hline & \multirow{2}{*}{$\begin{array}{l}\text { Total } \\
(n=139,519)\end{array}$} & \multicolumn{3}{|l|}{ Diabetes } \\
\hline & & No $(n=122,827)$ & Yes $(n=16,692)$ & p-value \\
\hline Age, years & & & & $<0.0001$ \\
\hline $40 \sim 64$ & $120,137(86.1)$ & $107,176(87.25)$ & 12,961 (77.63) & \\
\hline$\geq 65$ & 19,382 (13.9) & $15,651(12.75)$ & $3,731(22.37)$ & \\
\hline Smoking status & & & & $<0.0001$ \\
\hline Non & $53,894(44.53)$ & $47,189(44.26)$ & $6,705(46.52)$ & \\
\hline Ex & 11,244 (9.29) & $10,035(9.41)$ & $1,209(8.39)$ & \\
\hline Current & $55,892(46.17)$ & $49,392(46.33)$ & $6,500(45.10)$ & \\
\hline $\begin{array}{l}\text { Alcohol } \\
\text { consumption }\end{array}$ & & & & $<0.0001$ \\
\hline Non & $49,542(36.54)$ & $43,188(36.20)$ & $6,354(38.99)$ & \\
\hline $2 \sim 3 /$ month & $23,416(17.27)$ & $20,972(17.58)$ & $2,444(15.00)$ & \\
\hline$\geq 1$ /week & $62,637(46.19)$ & $55,139(46.22)$ & $7,498(46.01)$ & \\
\hline Exercise & & & & $<0.0001$ \\
\hline Non & $67,230(49.56)$ & $59,224(49.61)$ & $8,006(49.23)$ & \\
\hline 1 4/week & $55,119(40.63)$ & 49,091 (41.12) & $6,028(37.07)$ & \\
\hline$\geq 5$ /week & $13,298(9.8)$ & $11,069(9.27)$ & 2,229 (13.71) & \\
\hline $\begin{array}{l}\text { Household } \\
\text { income }\end{array}$ & & & & $<0.0001$ \\
\hline$<20 \%$ & $17,658(12.66)$ & $14,979(12.20)$ & $2,679(16.06)$ & \\
\hline Living place & & & & 0.679 \\
\hline Rural region & $76,126(54.60)$ & 66,997 (54.58) & $9,129(54.73)$ & \\
\hline Hypertension & $55,030(39.47)$ & $45,419(37.00)$ & $9,611(57.62)$ & $<0.0001$ \\
\hline Dyslipidemia & $22,358(16.04)$ & $17,866(14.56)$ & 4,492 (26.93) & $<0.0001$ \\
\hline BMI, kg/m² & $24.0 \pm 2.9$ & $24.0 \pm 2.9$ & $24.6 \pm 3.1$ & $<0.0001$ \\
\hline $\mathrm{TC}, \mathrm{mg} / \mathrm{dl}$ & $197.2 \pm 37.9$ & $196.9 \pm 36.8$ & $199.6 \pm 44.9$ & $<0.0001$ \\
\hline Glucose, mg/dl & $101.0 \pm 34.2$ & $92.9 \pm 12.8$ & $160.5 \pm 67.6$ & $<0.0001$ \\
\hline
\end{tabular}

Data are presented as the means $\pm \mathrm{SE}$, or $\%$ (SE).

BMI: body mass index, TC: total cholesterol, SE: standard error.

\section{The risk of prostate cancer increases with increasing BMI}

Table 2 shows regression analyses adjusted for age and other variables including smoking status, alcohol consumption, and exercise for prostate cancer development. Diabetes, dyslipidemia and BMI were remarkable risk factors. The HR for prostate cancer was stratified by BMI level in both age- and multivariable-adjusted models. The HR for prostate cancer was lowest in those with BMI $<18.5$ and highest for those with BMI $\geq 25$ in both models. Furthermore, there was a statistically significant increasing trend in risk of prostate cancer with increasing BMI in both models ( $\mathrm{p}<0.0001)$.

\section{The association between BMI and prostate cancer was influenced by the presence of diabetes}

Table 3 shows the HR for prostate cancer 
stratified by BMI according to the presence of diabetes in both age- and multivariable-adjusted models. Different associations between BMI and prostate cancer development were observed between the population with diabetes and the population without. In the population without diabetes, the HR for prostate cancer significantly increased as BMI increased beyond the reference BMI in a model adjusted for age and multiple variables (age, hypertension, dyslipidemia, smoking status, alcohol consumption, and exercise); however, the increase in HR was small. In the population with diabetes, the HR for prostate cancer increased significantly as BMI increased from $<18.5 \mathrm{~kg} / \mathrm{m}^{2}$ to within the reference range (18.5 to 22.9$)$ in the multivariable-adjusted model. In addition, a marked decrease in the HR was seen in those with BMI $<18.5 \mathrm{~kg} / \mathrm{m}^{2}$ compared to the reference or higher BMI population.

\section{Discussion}

The main findings of this population-based study are as follows: (1) men with a higher BMI were more likely to develop prostate cancer independent of confounding variables, (2) this positive association with prostate cancer and BMI is differentiated by existence of diabetes, and (3) in the population with diabetes, there showed a marked decreasing risk for prostate cancer in the underweight group compared with reference or higher weight group, unlike the population without diabetes.
Table 2. Age- and multivariable-adjusted hazard ratios for prostate cancer

\begin{tabular}{|c|c|c|c|}
\hline & & $\mathrm{HR}(95 \% \mathrm{CI})$ & p-value \\
\hline \multirow[t]{8}{*}{ Age-adjusted } & Diabetes & $\begin{array}{l}1.088(1.022, \\
1.158)\end{array}$ & 0.0085 \\
\hline & Hypertension & $\begin{array}{l}1.046(0.998, \\
1.095)\end{array}$ & 0.0585 \\
\hline & Dyslipidemia & $\begin{array}{l}1.106(1.042, \\
1.174)\end{array}$ & 0.0009 \\
\hline & BMI & & $<0.0001^{2}$ \\
\hline & $<18.5$ & $\begin{array}{l}0.863(0.753, \\
0.988)\end{array}$ & \\
\hline & $18.5-22.9$ & Ref. & \\
\hline & $23-24.9$ & $\begin{array}{l}1.088 \text { (1.027, } \\
1.153)\end{array}$ & \\
\hline & $\geq 25$ & $\begin{array}{l}1.195(1.132, \\
1.262)\end{array}$ & \\
\hline \multirow[t]{8}{*}{ Multivariable-adjusted ${ }^{1}$} & Diabetes & $\begin{array}{l}1.096(1.023, \\
1.174)\end{array}$ & 0.0093 \\
\hline & Hypertension & $\begin{array}{l}1.028(0.977, \\
1.081)\end{array}$ & 0.2959 \\
\hline & Dyslipidemia & $\begin{array}{l}1.088 \text { (1.019, } \\
1.161)\end{array}$ & 0.0117 \\
\hline & BMI & & $<0.0001^{2}$ \\
\hline & $<18.5$ & $\begin{array}{l}0.852(0.734, \\
0.99)\end{array}$ & \\
\hline & $18.5-22.9$ & Ref. & \\
\hline & $23-24.9$ & $\begin{array}{l}1.05(0.985, \\
1.119)\end{array}$ & \\
\hline & $\geq 25$ & $\begin{array}{l}1.133(1.067, \\
1.204)\end{array}$ & \\
\hline
\end{tabular}

Data are presented as HR (95\% confidence interval).

${ }^{1}$ Adjusted for age, smoking status, alcohol consumption, and exercise. ${ }^{2} p$ for trend.

HR: Hazard ratio, CI: confidence interval, BMI: body mass index.

Table 3. Age- and multivariable-adjusted hazard ratios for prostate cancer according to history of diabetes and body mass index, 2002-2012.

\begin{tabular}{|c|c|c|c|c|c|c|c|}
\hline \multirow[t]{2}{*}{ Diabetes } & \multirow[t]{2}{*}{ BMI } & \multirow[t]{2}{*}{ Total F/U } & \multirow[t]{2}{*}{ Incidence $^{1}$} & \multicolumn{2}{|c|}{ Age-adjusted } & \multicolumn{2}{|c|}{ Multivariable-adjusted } \\
\hline & & & & HR $(95 \%$ CI) & $\mathrm{P}^{3}$-value & HR (95\% CI) & $\mathrm{P}^{3}$-value \\
\hline \multirow[t]{5}{*}{ No } & $<18.5$ & $26,288.54$ & 8.14043 & $0.904(0.785,1.04)$ & & $0.886(0.758,1.035)$ & \\
\hline & $18.5-22.9$ & $358,832.28$ & 6.26755 & Ref. & & Ref & \\
\hline & $23.0-24.9$ & $291,638.14$ & 5.98344 & $1.076(1.101,1.145)$ & & $1.045(0.976,1.119)$ & \\
\hline & $\geq 25$ & $365,089.68$ & 6.1437 & $1.191(1.123,1.264)$ & & $1.125(1.052,1.202)$ & \\
\hline & & & & & $<0.0001$ & & 0.0008 \\
\hline \multirow[t]{5}{*}{ Yes } & $<18.5$ & $2,597.49$ & 5.00483 & $0.489(0.281,0.852)$ & & $0.535(0.3,0.953)$ & \\
\hline & $18.5-22.9$ & $39,047.58$ & 8.55367 & Ref. & & Ref. & \\
\hline & $23.0-24.9$ & $36,875.22$ & 8.84063 & $1.149(0.986,1.339)$ & & $1.069(0.903,1.265)$ & \\
\hline & $\geq 25$ & $59,116.01$ & 8.42411 & $1.183(1.028,1.361)$ & & $1.153(0.988,1.346)$ & \\
\hline & & & & & 0.0024 & & 0.0291 \\
\hline
\end{tabular}

Data are presented as HR (95\% confidence interval).

${ }^{1}$ All rates are expressed as number per 1000 person-years.

${ }^{2}$ Adjusted for age, hypertension, dyslipidemia, smoking status, alcohol consumption, and exercise.

${ }^{3} \mathrm{p}$ for trend

BMI: body mass index, HR: Hazard ratio, CI: confidence interval.

The underlying biological mechanism contributing to the epidemiologic positive association between obesity and prostate cancer can be partly explained by adipokine signaling, the insulin/insulin-like growth factor (IGF)-1 axis, and sex hormones [13, 16], all of which were mentioned frequently in a study regarding the association between obesity and prostate cancer. Briefly, adipose tissue itself is an important organ for the production of adipokines and inflammatory cytokines, which 
potentially arouse tumorigenesis and growth. So large adipose tissue deposits may lead to an increase of adipokines, mediated through a release from accumulated fatty tissue. In tandem with the accumulation of adipose tissue, obesity is linked with low-grade chronic subclinical inflammation with infiltrating macrophages, which stimulate the production of cytokines and pro-inflammatory factors involved in the pathogenesis of insulin resistance [16]. These changes can make pancreatic beta cells increase the production of insulin to maintain normal glucose levels, leading to hyperinsulinemia. Hyperinsulinemia has been shown to induce tumorigenesis in prostate cancer xenograft models $[17,18]$. Obesity and hyperinsulinemia promote increased circulating IGF-1, a recognized pathogenic role player in many cancers [13].

Another plausible mechanism linking obesity and prostate cancer is the change in sex hormone levels. Testosterone is aromatized to estradiol in adipose tissue. As the mass of adipose tissue increases, this aromatization pathway is upregulated, which leads to increased estradiol $[19,20]$, which is also involved in prostate cancer development and progression [21].

The cause of the differences in the association between prostate cancer and BMI in diabetic and non-diabetic men is not entirely clear, but might be partially explained by insulin secretion change related to differences in BMI.

Significant differences according to race in the association between diabetes and the development of prostate cancer have been seen in other studies [22]. One study was carried out with a Western population and provided evidence of an inverse association between diabetes and prostate cancer [18, 23]. On the other hand, another study strongly supported that diabetes is associated with an increased risk of prostate cancer in Asians [24]. The underlying pathophysiology of diabetes with prostate cancer is not fully understood. However, changes in the hormonal environment (androgens, insulin, IGF-1, leptin, etc.) of diabetic patients and superimposed genetic susceptibility might serve as at least a partial etiology [25]. Among the above changes, that of insulin is likely a key explanation for the inverse relationship between diabetes and prostate cancer.

Our results showed that the prostate cancer incidence between low and high BMI groups in diabetic patients was markedly different. It might be explained by the hormonal differences. Prostate cancer risk has been associated with hyperinsulinemia [26]. The weight gain can induce insulin resistance and result in hyperinsulinemia. Diabetic patients also might have more hormonal factors influencing the development of prostate cancer than non-diabetic patients, including variation in insulin level. In other words, in patients both with diabetes and obesity, these hormonal effects may be greater than in underweight diabetic patients through synergy. Consequently, in the underweight group of diabetic patients, prostate cancer incidence was markedly low compared to the other groups.

The distinctive feature of our study is that, to the best of our knowledge, it is the first population-based observational study on the relation between obesity and development of prostate cancer, stratified by the existence of diabetes. The findings of previous studies varied and showed conflicting results, with some finding obesity is a risk factor [27, 28], another reporting no association [29], and others showing an inverse relation between obesity and the development of prostate cancer [10, 30]. Because the diabetic population often has varying levels of circulating insulin, disease presence could be a possible confounding factor in analysis according hormone levels. This study showed a link between prostate cancer and BMI, with the positive relation varied according to the change of BMI category and the existence of diabetes.

The other distinctive feature of this study is that it was conducted on a region in which prostate specific antigen (PSA) screening is common and widespread. In South Korea, the cost of PSA is very low and covered by the national insurance service. Many people receive an annual medical checkup, which includes routine PSA screening. To explain conflicting results, the geographic differences related to PSA screening rates are suggested [31]. In a study conducted in Europe, where PSA screening is not as common as in the USA, there was a significant positive association between obesity and prostate cancer, whereas North American studies showed no association between obesity and prostate cancer. The different findings in the region where PSA screening is common and widespread are worthy of notice, because potential modifying factors of PSA screening are regulated.

One limitation of this study was that it did not include detailed biochemical information about the cancer stage and grade, and thus we could not evaluate the impact of those factors. The PSA level, digital rectal examination finding, family history, and medication history also were not included in this study. However, use of special medications such as statins and metformin were adjusted by disease diagnostic code such as E10 (diabetes) and E785 (dyslipidemia) instead. And the point that this study conducted in the region where PSA screening has been widespread could make up this limitation 
partially. Additionally, in Korea, the prostate cancer incidence has increased in the early 2000s. So the impact of family history was difficult to examine practically.

Another limitation was not differentiating between type I and type II diabetes. As mentioned above, diabetes type can have an influence on the level of circulating insulin. However, type II diabetes accounts for the majority of patients, and the proportion increases with age. Thus, this limitation would likely not influence our study outcomes.

\section{Conclusion}

This population-based study showed an association between obesity and development of prostate cancer that was influenced by BMI category and the presence of diabetes. Although the cause of these relationships remains unclear, our results suggest that different strategies for BMI control might be necessary to prevent prostate cancer in those with diabetes. Further research is needed to elucidate the effects of BMI on the development of prostate cancer in populations with and without diabetes.

\section{Abbreviations}

NHIS: National Health Insurance System; BMI: Body mass index; SE: standard error; HR: hazard ratio; CI: confidence interval; IGF: insulin/insulin-like growth factor; TC: total cholesterol; PSA: prostate specific antigen

\section{Acknowledgement}

The authors wish to acknowledge the financial support of the Catholic Medical Center Research Foundation made in the program year of 2016.

\section{Competing Interests}

The authors have declared that no competing interest exists.

\section{References}

1. Park SK, Sakoda LC, Kang D, et al. Rising prostate cancer rates in South Korea. Prostate. 2006; 66: 1285-91.

2. Chan JM, Holick CN, Leitzmann MF, et al. Diet after diagnosis and the risk of prostate cancer progression, recurrence, and death (United States). Cancer Causes Control. 2006; 17: 199-208.

3. Lichtenstein P, Holm NV, Verkasalo PK, et al. Environmental and heritable factors in the causation of cancer--analyses of cohorts of twins from Sweden, Denmark, and Finland. N Engl J Med. 2000; 343: 78-85.

4. MacInnis RJ, English DR. Body size and composition and prostate cancer risk: systematic review and meta-regression analysis. Cancer Causes Control. 2006; 17: 989-1003.

5. Renehan AG, Tyson M, Egger M, Heller RF, Zwahlen M. Body-mass index and incidence of cancer: a systematic review and meta-analysis of prospective observational studies. Lancet. 2008; 371: 569-78.

6. Freedland SJ, Platz EA. Obesity and prostate cancer: making sense out of apparently conflicting data. Epidemiol Rev. 2007; 29: 88-97.

7. Gong Z, Neuhouser ML, Goodman PJ, et al. Obesity, diabetes, and risk of prostate cancer: results from the prostate cancer prevention trial. Cancer Epidemiol Biomarkers Prev. 2006; 15: 1977-83.

8. Hsing AW, Sakoda LC, Chua S Jr. Obesity, metabolic syndrome, and prostate cancer. Am J Clin Nutr. 2007; 86: s843-57.
9. Rodriguez C, Freedland SJ, Deka A, et al. Body mass index, weight change, and risk of prostate cancer in the Cancer Prevention Study II Nutrition Cohort. Cancer Epidemiol Biomarkers Prev. 2007; 16: 63-9.

10. Wright ME, Chang SC, Schatzkin A, et al. Prospective study of adiposity and weight change in relation to prostate cancer incidence and mortality. Cancer. 2007; 109: 675-84.

11. Arnaldez FI, Helman LJ. Targeting the insulin growth factor receptor 1. Hematol Oncol Clin North Am. 2012; 26: 527-42, vii-viii.

12. Inzucchi SE, Matthews DR. Response to Comments on Inzucchi et al. Management of Hyperglycemia in Type 2 Diabetes, 2015: A Patient-Centered Approach. Update to a Position Statement of the American Diabetes Association and the European Association for the Study of Diabetes. Diabetes Care 2015;38:140-149. Diabetes Care. 2015; 38: e128-9.

13. Roberts DL, Dive C, Renehan AG. Biological mechanisms linking obesity and cancer risk: new perspectives. Annu Rev Med. 2010; 61: 301-16.

14. Yang Y, Yee D. Targeting insulin and insulin-like growth factor signaling in breast cancer. J Mammary Gland Biol Neoplasia. 2012; 17: 251-61.

15. World Health Organization Western Pacific Region. The Asia-Pacific perspective: redefining obesity and its treatment. Sydney, Australia: Health Communications Australia Pty Limited; 2000.

16. Waters KM, Henderson BE, Stram DO, Wan P, Kolonel LN, Haiman CA. Association of diabetes with prostate cancer risk in the multiethnic cohort. Am J Epidemiol. 2009; 169: 937-45.

17. Kasper JS, Liu Y, Giovannucci E. Diabetes mellitus and risk of prostate cancer in the health professionals follow-up study. Int J Cancer. 2009; 124: 1398-403.

18. Rodriguez C, Patel AV, Mondul AM, Jacobs EJ, Thun MJ, Calle EE. Diabetes and risk of prostate cancer in a prospective cohort of US men. Am J Epidemiol. 2005; 161: 147-52.

19. Carruba G. Estrogen and prostate cancer: an eclipsed truth in an androgen-dominated scenario. J Cell Biochem. 2007; 102: 899-911.

20. Williams G. Aromatase up-regulation, insulin and raised intracellular oestrogens in men, induce adiposity, metabolic syndrome and prostate disease, via aberrant ER-alpha and GPER signalling. Mol Cell Endocrinol. 2012; 351: 269-78.

21. Bonkhoff $\mathrm{H}$, Berges $\mathrm{R}$. The evolving role of oestrogens and their receptors in the development and progression of prostate cancer. Eur Urol. 2009; 55: 533-42.

22. Jian Gang P, Mo L, Lu Y, Runqi L, Xing Z. Diabetes mellitus and the risk of prostate cancer: an update and cumulative meta-analysis. Endocr Res. 2015; 40: 54-61.

23. Tsilidis KK, Allen NE, Appleby PN, et al. Diabetes mellitus and risk of prostate cancer in the European Prospective Investigation into Cancer and Nutrition. Int J Cancer. 2015; 136: 372-81.

24. Long XJ, Lin S, Sun YN, Zheng ZF. Diabetes mellitus and prostate cancer risk in Asian countries: a meta-analysis. Asian Pac J Cancer Prev. 2012; 13: 4097-100.

25. Rastmanesh R, Hejazi J, Marotta F, Hara N. Type 2 diabetes: a protective factor for prostate cancer? An overview of proposed mechanisms. Clin Genitourin Cancer. 2014; 12: 143-8.

26. Hsing AW, Chua S Jr, Gao YT, et al. Prostate cancer risk and serum levels of insulin and leptin: a population-based study. J Natl Cancer Inst. 2001; 93: 783-9.

27. Engeland A, Tretli S, Bjørge T. Height, body mass index, and prostate cancer: a follow-up of 950000 Norwegian men. Br J Cancer. 2003; 89: 1237-42.

28. Andersson SO, Wolk A, Bergström R, et al. Body size and prostate cancer: a 20-year follow-up study among 135006 Swedish construction workers. J Natl Cancer Inst. 1997; 89: 385-9.

29. Baillargeon J, Platz EA, Rose DP, et al. Obesity, adipokines, and prostate cancer in a prospective population-based study. Cancer Epidemiol Biomarkers Prev. 2006; 15: 1331-5.

30. Porter MP, Stanford JL. Obesity and the risk of prostate cancer. Prostate. 2005; 62: 316-21.

31. Allott EH, Masko EM, Freedland SJ. Obesity and prostate cancer: weighing the evidence. Eur Urol. 2013; 63: 800-9. 